\title{
Erratum to: Bariatric Surgery and Endoluminal Procedures: IFSO Worldwide Survey 2014
}

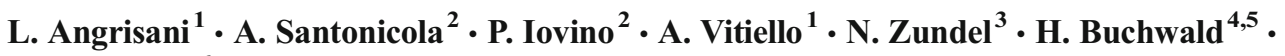 \\ N. Scopinaro ${ }^{6}$
}

Published online: 5 July 2017

(C) Springer Science+Business Media, LLC 2017

\section{Erratum to: OBES SURG \\ DOI 10.1007/s11695-017-2666-x}

In Table 4, which reports numbers of different procedures in each country, column headings for Gastric Plication (GP) and One Anastomosis Gastric Bypass (OAGB) have been interchanged. Despite this error, we want to reassure the reader that all the numbers have been double-checked and the results are correct. All the other tables and charts have not been influenced by this mistake. Numbers and statistics of OAGB

The online version of the original article can be found at http://dx.doi.org/ 10.1007/s11695-017-2666-x

A. Santonicola

antonellasantonicola83@gmail.com

H. Buchwald

buchw001@umn.edu

N. Scopinaro

nicola.scopinaro@gmail.com

1 General and Endoscopic Surgery Unit, S. Giovanni Bosco Hospital, Naples, Italy

2 Gastrointestinal Unit, Department of Medicine and Surgery, University of Salerno, Via S. Allende, Baronissi, Salerno, Italy

3 Minimally Invasive and Bariatric Surgery, FSFB, Bogota, Colombia

4 Department of Surgery, University of Minnesota, Minneapolis, MN 55455, USA

5 Department of Biomedical Engineering, University of Minnesota, Minneapolis, MN 55455, USA

6 Department of Surgery, School of Medicine, University of Genoa, Genoa, Italy worldwide and in the IFSO chapters have not been altered.

In Table 5, please note that the figures of the populations refer to the adult population (15-64 years) eligible for bariatric and metabolic surgery (source: http://www. indexmundi.com/it/).

This erratum also gives us the opportunity to sincerely express our gratitude to the presidents of the IFSO National Societies, who have worked hard to collect data from their registries. The corrected Table 4 follows. 
Table 4 The bariatric/metabolic surgical procedures and the endoluminal procedures performed in the four IFSO regions of the world: USA/Canada, Europe, Latin/South America, and Asia/Pacific

\begin{tabular}{|c|c|c|c|c|c|c|c|c|c|}
\hline Country & Total & AGB & RYGB & SG & $\mathrm{BPD} / \mathrm{DS}$ & OAGB & GP & Other & $\begin{array}{l}\text { Endolum } \\
\text { procec }\end{array}$ \\
\hline \multicolumn{10}{|l|}{ North America } \\
\hline Canada & 6.522 & 702 & 3.158 & 2.362 & 300 & 0 & 0 & 0 & 0 \\
\hline USA & 191.920 & 18.500 & 59.124 & 113.381 & 886 & 0 & 29 & 0 & 0 \\
\hline Total per area & 198.442 & 19.202 & 62.282 & 115.743 & 1.186 & 0 & 29 & 0 & 0 \\
\hline \multicolumn{10}{|l|}{ Europe } \\
\hline Austria & 2.553 & 91 & 1.418 & 521 & 4 & 496 & 0 & 23 & 18 \\
\hline Azerbaijan & 16 & 0 & 1 & 14 & 0 & 1 & 0 & 0 & \\
\hline Belgium & 12.000 & 1.000 & 5.500 & 4.000 & 0 & 1.500 & 0 & 0 & \\
\hline Czech Republic & 1.448 & 280 & 90 & 150 & 50 & 8 & 670 & 200 & 220 \\
\hline Egypt & 10.340 & 180 & 1.500 & 3.100 & 40 & 800 & 200 & 4.520 & 1200 \\
\hline Finland & 839 & 0 & 694 & 139 & 4 & 0 & 0 & 2 & \\
\hline France & 46.960 & 4.364 & 14.015 & 28.581 & 0 & 0 & 0 & 0 & \\
\hline Germany & 7.298 & 133 & 3.332 & 3.681 & 9 & 131 & 9 & 3 & \\
\hline Greece & 1.327 & 110 & 85 & 756 & 8 & 275 & 65 & 28 & 10 \\
\hline Iceland & 163 & 108 & 52 & 3 & 0 & 0 & 0 & 0 & \\
\hline Israel & 8881 & 659 & 877 & 7262 & 65 & & 0 & 18 & \\
\hline Italy & 8867 & 2182 & 1628 & 3799 & 124 & 477 & 268 & 389 & \\
\hline Lebanon & 0 & & & & 0 & & & 0 & \\
\hline Lithuania & 252 & 63 & 103 & 6 & 0 & 0 & 68 & 12 & 12 \\
\hline Netherlands & 8350 & 77 & 6757 & 1158 & 10 & 44 & 0 & 304 & 80 \\
\hline Norway & 3002 & & 1653 & 1316 & 30 & 3 & 0 & 0 & \\
\hline Poland & 2531 & 318 & 492 & 1334 & 1 & 207 & 0 & 179 & 179 \\
\hline Portugal & 2892 & 94 & 1290 & 986 & 54 & 260 & 9 & 199 & \\
\hline Romania & 1380 & 27 & 99 & 1128 & 11 & 22 & 39 & 54 & 17 \\
\hline Russian Federation & 1621 & 419 & 118 & 861 & 71 & 29 & 7 & 116 & 77 \\
\hline Serbia & 0 & 0 & 0 & 0 & 0 & 0 & 0 & 0 & 0 \\
\hline Slovenia & 200 & 3 & 31 & 26 & 0 & 115 & 0 & 25 & \\
\hline South Africa & 566 & 0 & 423 & 63 & 68 & 0 & 0 & 12 & \\
\hline Spain & 4030 & 126 & 1562 & 1839 & 142 & 34 & 27 & 300 & \\
\hline Sweden & 6607 & 2 & 5386 & 1090 & 47 & 0 & 4 & 78 & 39 \\
\hline Switzerland & 4167 & 17 & 3173 & 646 & 41 & 17 & 0 & 273 & \\
\hline Turkey & 6347 & 200 & 1350 & 3520 & 125 & 818 & 60 & 274 & 100 \\
\hline Ukraine & 251 & 2 & 30 & 91 & 47 & 13 & 27 & 41 & 18 \\
\hline United Kingdom & 6391 & 823 & 3011 & 2012 & 13 & 0 & 0 & 532 & 198 \\
\hline Total per area & 149.279 & 11.278 & 54.670 & 68.082 & 964 & 5.250 & 1.453 & 7.582 & 2.168 \\
\hline \multicolumn{10}{|l|}{ Latin America } \\
\hline Argentina & 36.668 & 0 & 25.520 & 8.754 & 2.227 & 35 & 0 & 132 & 132 \\
\hline Bolivia & 314 & 0 & 92 & 174 & 0 & 2 & 36 & 10 & \\
\hline Brazil & 97.480 & 450 & 66.000 & 20.200 & 1.050 & 600 & 30 & 9.150 & 8600 \\
\hline Chile & 5.311 & 3 & 1.133 & 3.814 & 0 & 0 & 3 & 358 & 196 \\
\hline Colombia & 12.700 & 4.800 & 7.200 & 0 & 50 & 150 & 0 & 500 & 500 \\
\hline Costa Rica & 1.448 & 280 & 90 & 150 & 50 & 8 & 670 & 200 & 220 \\
\hline Dominican Republic & 1.117 & 0 & 29 & 981 & 7 & 38 & 20 & 42 & 8 \\
\hline Ecuador & 626 & 0 & 80 & 150 & 3 & 40 & 10 & 343 & 30 \\
\hline Guatemala & 200 & 2 & 68 & 112 & 0 & 0 & 8 & 10 & 10 \\
\hline Mexico & 0 & & & 0 & & & & 0 & \\
\hline
\end{tabular}




\begin{tabular}{|c|c|c|c|c|c|c|c|c|c|}
\hline Panama & 0 & & & & 0 & & & 0 & \\
\hline Paraguay & 300 & 0 & 300 & 0 & 0 & 0 & 0 & 0 & \\
\hline Perù & 0 & & & & 0 & & & 0 & \\
\hline Venezuela & 4.472 & 11 & 2.880 & 1.498 & 3 & 0 & 0 & 80 & \\
\hline Total per area & 160.636 & 5.546 & 103.392 & 35.833 & 3.390 & 873 & 777 & 10.825 & 9.696 \\
\hline \multicolumn{10}{|l|}{ Asia/Pacific } \\
\hline Australia-New Zealand & 15.136 & 3.604 & 1.019 & 10.227 & 31 & & & 255 & \\
\hline China & 4.195 & 50 & 1.866 & 2.229 & 0 & 0 & 20 & 30 & \\
\hline Hong Kong & 144 & 2 & 4 & 116 & 1 & 1 & 1 & 19 & 17 \\
\hline India & 11.336 & 12 & 1.833 & 7.638 & 28 & 1.537 & 12 & 276 & 22 \\
\hline Japan & 222 & 4 & 20 & 144 & 54 & 0 & 0 & 0 & \\
\hline Korea & 889 & 439 & 134 & 166 & 0 & 0 & 43 & 107 & \\
\hline Kuwait & 5.498 & 244 & 61 & 3.803 & 4 & 22 & 0 & 1.364 & 1356 \\
\hline Saudi Arabia & 15.571 & 1.215 & 3.033 & 8.649 & 500 & 1.580 & 300 & 294 & 294 \\
\hline Singapore & 299 & 2 & 103 & 193 & 0 & 0 & 0 & 1 & 1 \\
\hline Taiwan & 2.421 & 31 & 119 & 1.484 & 0 & 194 & 21 & 572 & 34 \\
\hline UAE & 4033 & 170 & 262 & 3223 & 0 & 230 & 0 & 148 & 87 \\
\hline Total per area & 59.744 & 5.773 & 8.454 & 37.872 & 618 & 3.564 & 397 & 3.066 & 1.811 \\
\hline
\end{tabular}

